\title{
Towards the Second Edition of the Book "QSAR-Mapping and SBGN-Mapping for Biological Samples (Lecture Course and Special Practicum)"
}

\section{Editorial Column}

\section{O.V. Gradov}

I am very glad to present to the readers of "Medical Imaging Process \& Technology" journal the forthcoming second edition of our book "QSAR-mapping and SBGN-mapping for Biological Samples (Lecture Course and Special Practicum)", which is actually an introduction to the novel microscopic imaging approaches for numerous biomedical applications. It is possible to imagine that imaging and morphology of biological structures is not a rigorous mapping of different "material points" and structures in the field of view (or ROI's), because the living state of the sample is a complex of many spatiotemporal dynamic processes. But it is much more difficult to imagine that such spatiotemporal dynamic processes also can be mapped on the temporal scales as a function of their compartmentalization in the cellular media, and consequently, that the map of the physiological-metabolic state from the cell level to the organism level can be visualized or interpreted not only as a map of localizations and colocalizations of different metabolic agents, but also as a map of their activities (and colocalizations or spatiotemporal correlations of the agent-based kinetics) in the ultra-structural and / or intercellular / multi-cellular processes. It is well known, that the activities of the biochemical agents can be described within the framework of the SAR approach, in particular, in Quantitative Structure-Activity Relationship (QSAR) models. Consequently, the basic problem of the agent-based representation and spatiotemporal imaging of the metabolic functions is the problem of the morphism map (or functor map) between the activities' / properties' sets and the structure set (as a surjection) in the QSAR/QSPR projection on the map of the biological sample which can be formed using standard technologies of contemporary microscopy and multi-/ hyper-spectral imaging techniques (including non-optical ranges of wavelengths or frequencies of the analytical signals and sub-wavelength superresolution physical imaging principles of the biostructure visualization). The second problem of the descriptometric / descriptographic mapping using QSAR/QSPR projections of molecular data sets on the microscopic optical (or non-optical) surface or profile

Copyright $\odot 2018$ O.V. Gradov

doi: $10.24294 /$ mipt.v1i2.1001

EnPress Publisher LLC.This work is licensed under the Creative Commons Attribution-NonCommercial 4.0 International License (CC BY-NC 4.0). http://creativecommons.org/licenses/by/4.0/ 
of the biological sample or the living object is a problem of interrelationship morphism ${ }^{1}$ mappings or functor-assisted mappings between the QSAR map, QSPR map and SBGN map (System Biology Graphical Notations) on the real dynamical map of the biological structure. Consequently, the way of the comprehensive multiparametric mappings of the biological structures is an algorithm of transforms between the corresponding data types on the $2 \mathrm{D}$ or $3 \mathrm{D}$ coordinate meshes with the kinetic-oriented temporal resolution.

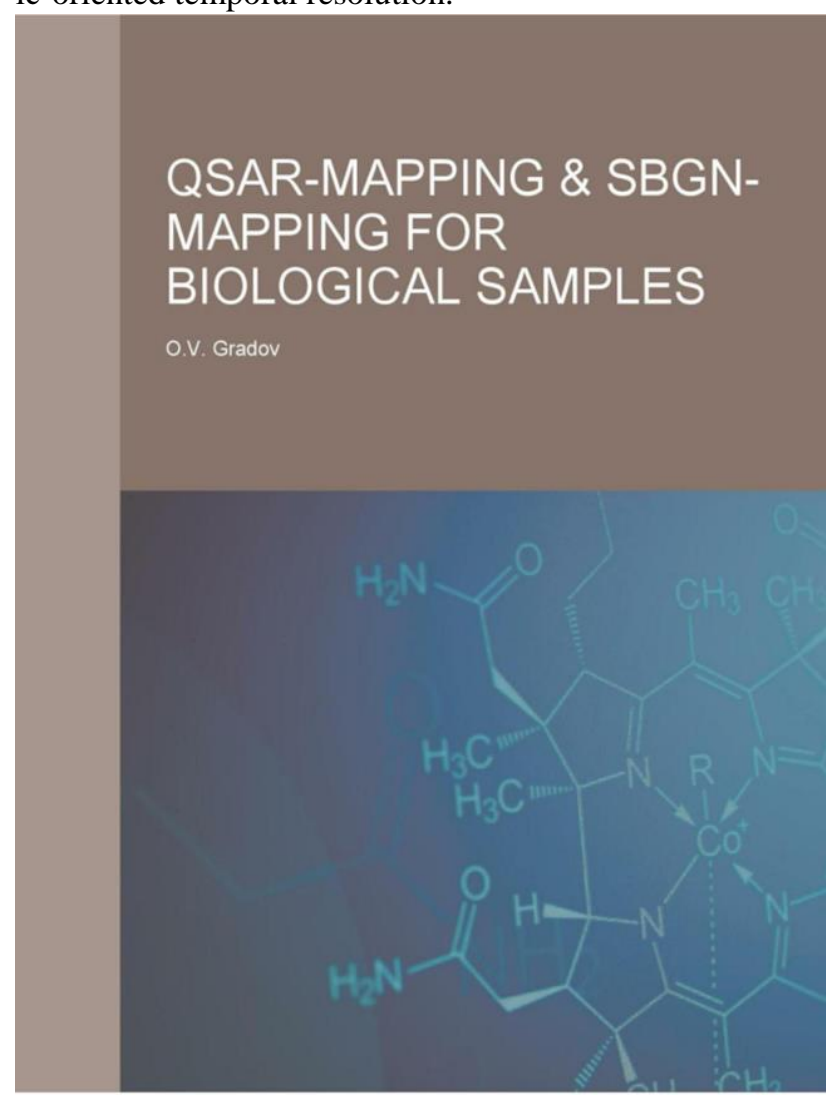

Figure 1; The first edition of the book "QSAR-mapping and SBGN-mapping for biological samples (Lecture

1 In mathematics, a morphism is a structure-preserving map from one mathematical structure to another one of the same type. The notion of morphism recurs in much of contemporary mathematics. In set theory, morphisms are functions; in linear algebra, linear transformations; in group theory, group homomorphisms; in topology, continuous functions, and so on. In category theory, morphism is a broadly similar idea, but somewhat more abstract: the mathematical objects involved need not be sets, and the relationship between them may be something more general than a map, although has to behave similarly to maps, e.g. has to admit associative composition (Remark from the Technical Editor of the Monograph).
Course and Special Practicum)" (New York, Academic Pub. - XanEdu, 2015; Author: O.V. Gradov, INEPCP RAS).

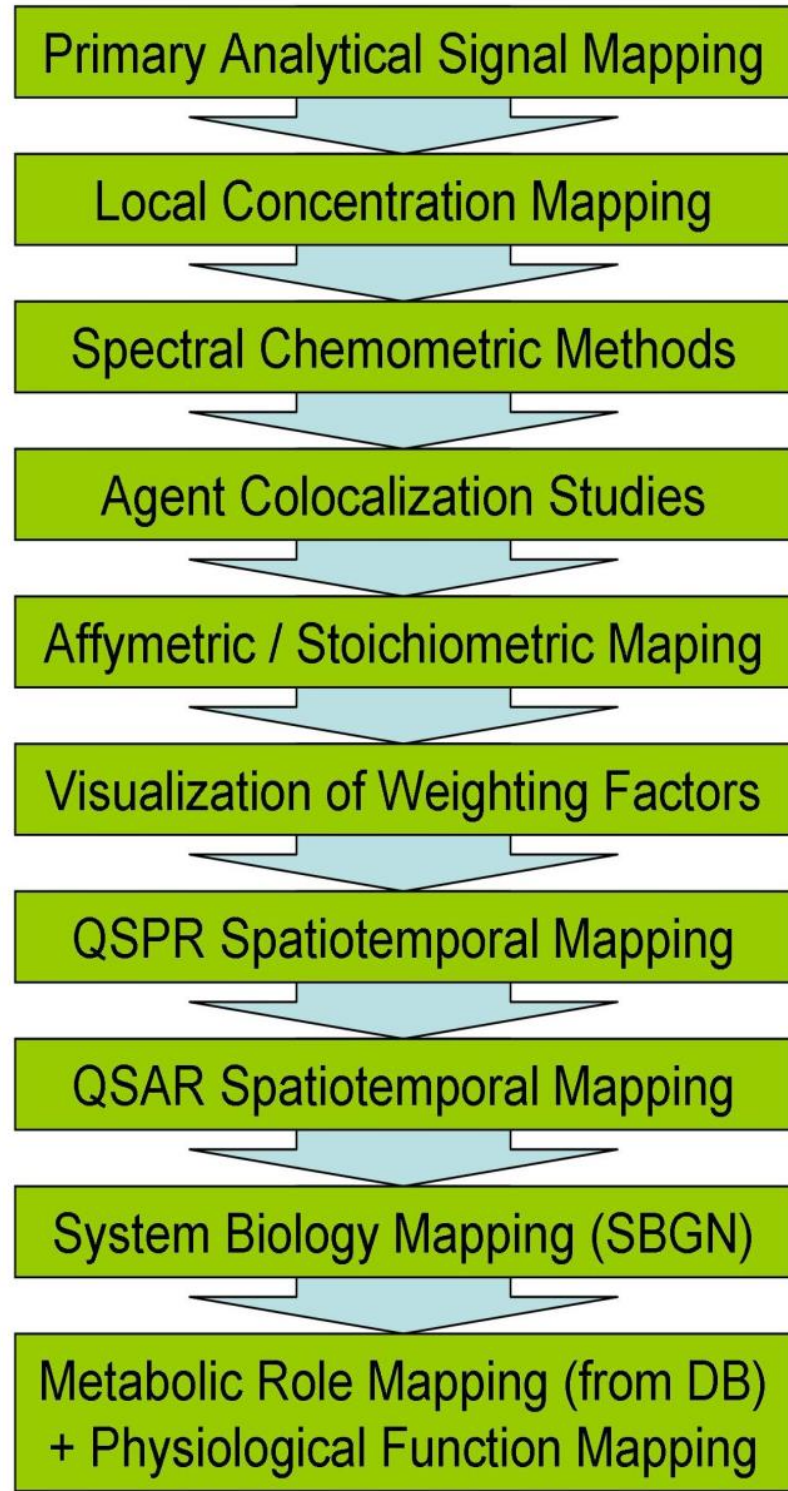

Figure 2; The algorithm of QSAR/QSPR- and SBGN-mapping.

In the first edition of the annotated book ${ }^{[1]}$ (Figure 1) we proposed a way to resolve such problems. The basic algorithm for hybridization between the QSAR-mapping, QSPR-mapping and SBGN-mapping is shown in Figure 2. It includes the following procedures:

a) Primary Analytical Signal Mapping (PAMS). This procedure is partially equivalent to the microspectrophotometric or cytospectrophotometric techniques in the standard microscopic systems. It is also similar to the quantitative multi-spectral imaging or quantitative hyper-spectral imaging in some cases with the possibility of 
"multi-layer colocalization" analysis at different wavelengths.

b) Local Concentration Mapping (LCM). The process of decoding of the microspectrophotometric / microspectrometric data or convertion of the fluorescence or extinction parameters into the concentration maps, digital data arrays and spatiotemporal kinetic distribution profiles (or S[T]KDP's) is the LCM procedure.

c) Spectral Chemometric Methods. Multivariate calibration in the framework of the multivariate models is a technique, providing a possibility to measure the multi-wavelength spectral response to the analyte concentrations in the biological samples. If we have more than one agent with more than one spectral distribution or S[T]KDP in the sample, it is obvious that the process of the local point-to-point multivariate calibration for all the components is strongly requred.

d) Agent Colocalization Studies. After the spectrochemometric procedure application to the sample (as the comprehensive complex of analytes), it is rational to investigate the spatiotemporal structure of the localized kinetic trends of the compartmentalized sample. It is very interesting and heuristically valuable, because the chemical nature of the interactions between the bioactive molecules can be described in terms of activation and inhibition, mutually effects and antagonistic cross-(re)activities / competitive interactions. It is possible that the time bin or interval for different processes can be simultaneously characterized as the interval for increasing of concentrations for some components and as the decreasing concentration interval for the others.

e) Affymetric / Stoichiometric Maping. As a primary consequent of the above listed pecularities of the "biokinetik dialectics", one can conclude, that the nature of such interactions is in fact the nature of reversible and irreversible processes of partially stoichiometric /partially "affymetric" interactions with different stabilities of the covalent structures and non-covalent coordination complexes, which can be provided by different mechanisms of the chemical structure formation (and consequently, with different mechanisms of their activities in the framework of QSAR principles). The map of the stoichiometric reactions (or S[T]KDP) and supramolecular coordination fixation processes (or their $\mathrm{S}[\mathrm{T}] \mathrm{KDP}$ 's) can be formatted as a carrier map for the
QSAR pattern map. It is partially equivalent to the hybridized ratiometric (not absolute, but ratio-based estimation of the physical and chemical effects, i.e. stoichiometry or the multi-agent affinity ratios) and ratemetric (i.e. kinetic act counting) approaches, proposed by the author's group in 2014-2016 ${ }^{[2,3]}$.

f) Visualization of Weighting Factors (VWF). VWF's can be annotated as the functions of the cross-channel (or multilayer - for multi-spectral and hyper-spectral imaging) normalization not by the criterion of the chemical equilibrium (in contrast to the fact that multivariate calibration of the multi-wavelength spectral response to the analyte concentrations in the framework of the chemometrical approaches also can be interpreted as a cross-channel normalization and weight factor optimization), but only as the weight functions of the activity in the cell or in the organism (and also in the special cases as a normalization to the biogeochemical "clarcks" of the substance).

g) QSPR Spatiotemporal Mapping (QSPR-SM). Quantitative structure-property relationship (QSPR) is a SAR variant when a chemical property is modeled as the response variable. This is a good "projection" between the analytical procedure characteristics / algorithms and the results of the analytical procedure implementation (COBAC-assisted measurements) in the framework of the possible "data mining" for this type of analytical procedures. In the classical article by Yousefinejad and Hemmateenejad one can find a good explication from the history: "properties or behaviors of chemical molecules have been investigated in the field of QSPR. Some examples are quantitative structure-reactivity relationships (QSRRs), quantitative structure-chromatography relationships (QSCRs) and ... quantitative structureelectrochemistry relationships (QSERs), and quantitative structure-biodegradability relationships (QSBRs)" ${ }^{\text {"[4] }}$.

h) QSAR Spatiotemporal Mapping (QSAR-SM). Quantitative structure-activity relationship models are regression and classification models used in the biochemical and biomedical sciences, including biomedical diagnostic spectroscopy and medical diagnostic imaging. "A QSAR has the form of a mathematical model: activity $=\mathrm{f}($ physiochemical properties and/or structural properties) + error". This obvious formulation does not consider the quantitative and qualitative difference between 
the biological, chemical and physical errors. For this reason, we have introduced the stages $\mathrm{C}$ and $\mathrm{F}$ for comparative qualimetric analysis in the GUI before the stages G and H. QSAR and QSPR maps are not the intermediate or transitive results of the research, but they are basic layers and maps with the conformed coordinate meshes for SBGN data pull projections on the sample morphology layer (see Fig. 3).

i) SBGN Mapping / System Biology Mapping. It is the final metrological stage of this research algorithm implementation. If we have a QSAR or QSPR map of the organism with one-to-one mapping of it's morphology, it is obvious, that the metabolic pathway structure can be decoded or deciphered using SBGN key or scheme of the processes, including "Process Descriptions" ("PD"), "Entity Relationships" ("ER") and "Activity Flows" ("AF") for the indicated chemical structures on the morphological map of the object.

j) Metabolic Role Mapping and Physiological Function Mapping. It is noteworthy, that SBGN data obtained may not be equivalent to the decoding of function if we have no data about the chemical agents and their physiological roles in the organism. Consequently, we have a final resolving stage for our sophisticated data as a non-formal agent description using diagnostic databases "in language" (for classical medical biochemists it can be provided using metabolic or clinical variable descriptors, for (bio)medical physiologists it can be implemented using databases of (cyto)physiological functions and / or their anatomical localizations in the human body).

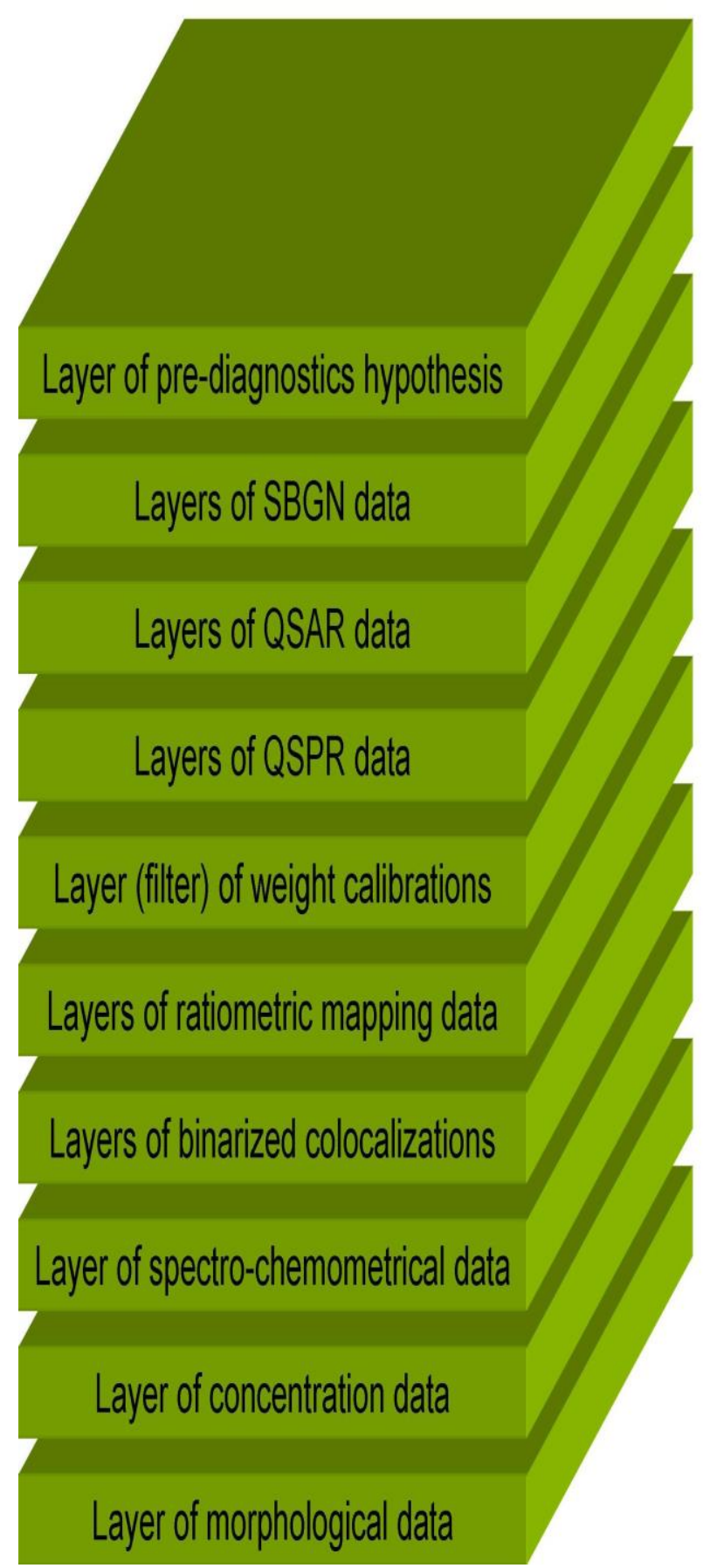

Figure 3; The multi-dimensional image of colocalized descriptors in frame of QSAR/QSPR+SBGN-concept.

Therefore, the resulting multi-dimensional images or the sample patterns can be represented in the way, schematically shown in Figure 3 as a.) multi-layer structure (not equivalent to the spatial decomposition for the $3 \mathrm{D}$ structure rendering, such as "confocal image binarization" patterns for "Imaris"-like software).

\section{References}

1. Gradov OV. QSAR-mapping and SBGN-mapping 
for biological samples (Lecture Course and Special Practicum). New York, Academic Pub. Ambassador Education Solutions, 2015. DOI:

10.13140/RG.2.1.4452.2082

2. Orehov TC, Gradov OV. [Hybridization of COBAC, QSPR / QSAR and SBGN technologies: The unity of theory and practice for biomedical technique design and biochemical diagnostic information analysis] // [Biotechnosphere]. 2014; 33(3): 29-31 [in Russian].

3. Orehov TC, Gradov OV (2016). Hybridization of
COBAC, QSPR / QSAR and SBGN technologies: The unity of theory and practice for biomedical technique design and biochemical diagnostic information analysis. Journ. Med. Bioeng. 2016; 5(2): 128-132. DOI: 10.18178/jomb.5.2.128-132

4. Yousefinejad S, Hemmateenejad B. Chemometrics tools in QSAR/QSPR studies: A historical perspective. Chemometrics and Intelligent Laboratory Systems. 2015; 149, Part B: 177-204. DOI:10.1016/j.chemolab.2015.06.016. 